Prog.Neuro-Psychopharmacol. Vol.4,pp.37-41, 1980.

Pergamon Press Ltd.Printed in Great Britain.

\title{
LFHIUM AND THE STRUCTURE OF EXPLORATORY BEHAVIOR IN THE RAT
}

\author{
Richard J. Katz \\ Mental Health Research Insitute, Department of Psychiatry \\ University of Michigan Medical Center, Ann Arbor, Michigan 48109, USA
}

(Final form, December 1979)

\begin{abstract}
1. Adult male Sprague Dawley rats were given lithium as a dietary supplement, or a control diet.

2. A single seven hour test of exploratory behavior was carried out after one week of chronic drug exposure.

3. The structure of exploration was significantly altered by the diet, with experimental animals exploring less intensely and with increased spacing of activity episodes.

4. These findings may represent a useful preclinical analog of some typically observed therapeutic effects of lithium.
\end{abstract}

Keywords: biological rhythms, exploratory activity, lithium, motor-behavior

\section{Introduction}

The alkali metal lithium remains a drug of choice in the treatment of a variety of psychiatric disorders, particularly bipolar affective disorders, acute mania, and diverse other syndromes characterized by a recurrent and episodic nature (Gershon and Shopsin, 1973). A number of laboratory models have been used in investigating lithium's pharmacological mode of action. One class of model has concentrated upon psychomotor activation as an analog of clinical hyperactivity (Carroll and Sharp, 1971; Cox et al., 1971; Johnson, 1972, 1976; Mukherjee et al., 1977; Smith and Smith, 1973; Smith, 1975). These and related reports (Gray et al., 1976; Syme and Syme, 1973) have suggested that lithium selectively decreases rearing activity (Gray et al., 1976; Johnson, 1972; Wolthius et al., 1975) or open field activity (Smith and Smith, 1973; Syme and Syme, 1973) although these reports are not consistent regarding the relative proportions to which the behaviors are reduced by treatment. Other reports, moreover, have occasionally noted only minor changes in spontaneous activity with chronic treatment (D'Encarnacao and Anderson, 1970; Segal., et al 1975). The majority of reports, however, agrees that chronic lithium treatment does reduce some aspects of exploratory behavior.

A second class of model has investigated lithium's effects upon biological rhythms (Engelmann, 1973; Engelmann et al., 1974). It has been suggested that lithium alters the occurrence of biological rhythms and that its effects upon oscillating systems may be important in its clinical efficacy. The typical preparation used for the study of these effects unfortunately is a 
plant (Kalanchoe blossfeldiana), although limited research has also involved rodents. There is considerable utility in further investigating related effects using mammalian behavior as a dependent variable.

One easily overlooked aspect of exploratory behavior is its episodic orbanization, particularly during extended tests. For example, rats placed in novel environments generally are active for thirty to sixty minutes. They then are quiescent for an extended period, following which they again are active. Activity and quiescence continue to oscillate over the testing period. It follows that exploratory behavior tested over an extended session has many of the requisite properties for simultanaously inodelling both heightened activity and recurrent events with an episodic character. We have utilized these characteristics of extended exploration in further investigating the behavioral pharmacology of lithium. The present design both replicated and extended previous models of this drug's effects upon behavior, utilizing a novel model of recurrent exploratory activity.

\section{Methods}

Subjects: Subjects were sixteen experimentally naive adult inale Sprague Dawley rats $(250-300 \mathrm{~g}$ : eight/group) obtained localy (Charles River Farms; Portage, MI) and maintained on ad libitum food and tap water. Rats were housed four/cage in $36 \times 33 \times 17 \mathrm{~cm}$ polypropylene containers, and lighting cycles of $12 \mathrm{~h} / 12 \mathrm{~h}$ were automatically programmed (lights on - 8:00-20:00).

Drug: To insure continual exposure to lithium and to more closely approximate patterns of clinical ingestion the drug was administered in the normal diet of the experimental animals (as lithium chloride $120 \mathrm{mEq} / \mathrm{Kg}$ of Teklad $4 \%$ fat rodent diet S0836). To compensate for normal ionic loss which occurs during Iithium treatment supplements of sodium and potassium chloride ( 1100 and $100 \mathrm{mEq}$ respectively) were also added to the food, as recommended by olesen et al. (1975). The effective daily dose range for the present experiment was $.3-.7 \mathrm{mEq}$. based upon records of food consumption.

Procedure: At 9:00 h of the test day rats were removed from group housing and individually placed in test cages containing a novel bedding (pinewood sawdust). Cage shape, bedding, and individual exposure were therefore all novel for the subjects. Subjects remained undisturbed on the platforms for seven hours, and were then returned to their home cages. Data were collected as ten minute blocks. Lithium treated subjects were maintained for one additional day of drug exposure and then sacrificed (between $11: 00-12: 00 \mathrm{~h})$ by decapitation, bloods immediately were collected in heparinized tubes and centrifuged for determination of plasta lithium levels.

Statistical Analysis: All data are presented as median scores (table one) or untransformed individual scores (figure one). Due to large variability across subjects all data were analyzed nonparametrically by the Kolmogorov-Smirnov statistic and the Mann Whitney U-test (Siegel, 1956). To standardize comparisons an episode of activity was defined as such only if it contained greater than 50 counts $/ 10 \mathrm{~min}$. Subthreshold activity was considered behavioral quiescence.

Apparatus: The testing apparatus consisted of a $51 \times 41 \times 22$ cm white polypropylene cage. Four cages were used at any time, and were placed upon commercially available field sensitive activity monitors (Stoelting, Chicago, Ill.) operating on a selective mode for the detection of gross body activity. Monitors were adjusted to within 5\% sensitivity of each other, and care was taken to counterbalance treatment groups across monitors. The testing environment was illuminated by overhead fluorescent ( $70 w$ ) bulbs, and the normal operation of air circulating equipment provided 25 dB masking noise. Additional details of the platforms have been presented elsewhere (Katz, et al., 1978). Plasma lithium was determined photometrically using a Perkin-Elmer 305-B atomic absorption spectrophotometer. 
Table 1

Effects of lithium on exploratory activity in rats

\begin{tabular}{lrrrrrrrrrr}
\hline Min & 10 & 20 & 30 & 40 & 50 & 60 & 70 & 80 & 90 & 100 \\
Lithium & 542 & 635 & 277 & 43 & 0 & 0 & 0 & 4 & 4 & 1 \\
Control & 860 & 752 & 484 & 307 & 104 & 25 & 22 & 10 & 4 & 16
\end{tabular}

$\bar{N}=8$ each, group median values

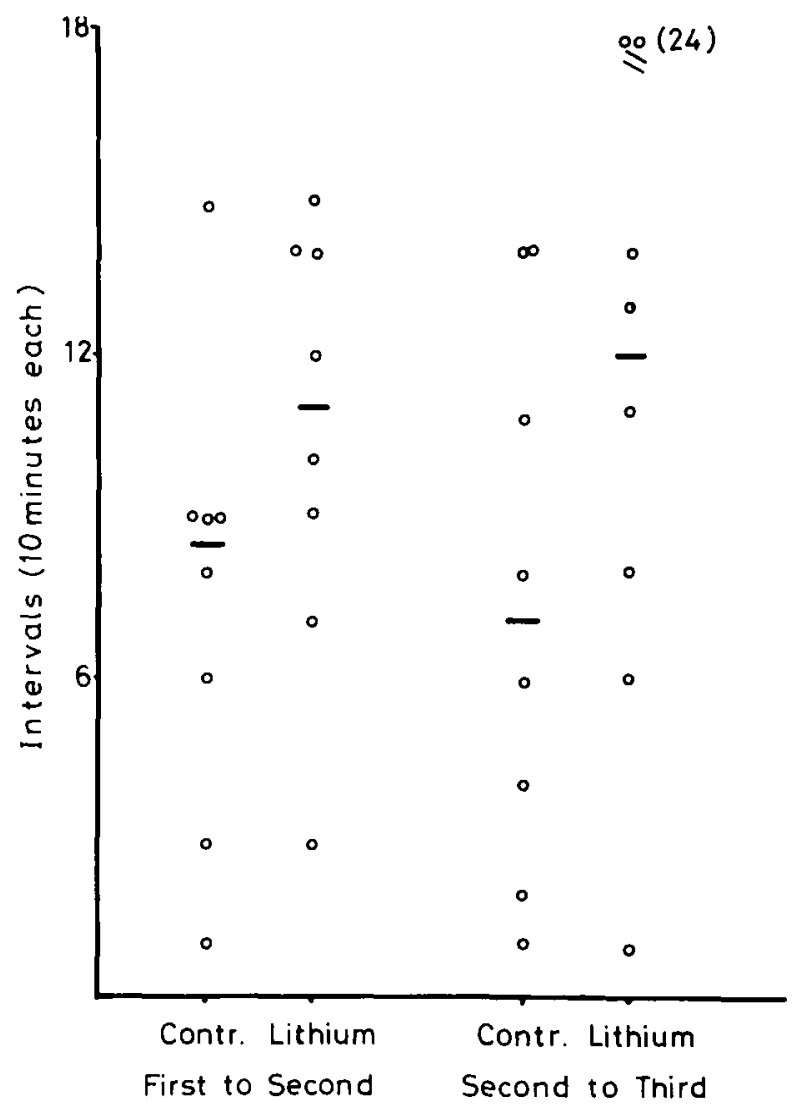

Figure 1. Lithium induced alteration in the spacing of exploratory episodes. Lithiun or control diets were administered chronically for seven days prior to placement in activity cages for a single exposure of seven hours.

\section{Results}

Lithium produced a lowering of exploration and a greater spacing of exploratory episodes. Table 1 presents the first ten recording intervals which include the initial exploratory episode for both experimental and control groups. 
Table one shows that lithium produced a lowering of both the intensity and duration of a typical initial exploratory episode. Other results suzgest it also altered the typical profile of activity over time (figure one). When number of ten minute blocks to subsequent episode (i.e. inter-episode duration) was compared across groups the median number for the lithium treated group was 11 (range = 3-15) while the median contol difference was 8.5 ( $r a n g e$ $=1-15)$. This difference was statistically reliable $(U=10.5, p<02)$.

A similar pattern held for the inter-episode length between second and third episodes, with a median score for lithium animals of 12 (range 1-24) and for control animals of 7 (range $=1-14$ ). This last difference however was not reliable $(U=34, p \sim .5)$.

Finally, examination of number of episodes/session for the two zroups indicated lithium treated subjects nas fewer (Median $=3 ;$ range $=2-5$ ) episodes than control subjects (median $=4 ;$ range $=3-5)(U=16 ; \quad p<.05$ by median test. Mean plasma lithium value for the experimental group was . $8 \mathrm{mEq}$ (range $=.3-1.2)$.

\section{Discussion}

Lithium reduced initial and subsequent patterns of exploration in chronically treated rats. These changes reflect both an initial lowering of activity and a re-organization of exploratory behavior over time, with a lengthening of episodes and a reduction in total episodes. The present results confirm earlier studies suggesting that lithium reduces exploration, and also suggest several novel parameters of exploratory behavior were affected. It might be noted that while lithium effects persisted throughout the testing interval there apparently was a decline in the degree to which they were present. The reason for this may rest with experimental testing procedures. All subjects were tested after their normal feeding cycle. However testing continued over an extended period and it might be anticipated that drug levels would therefore decline over time. As drug levels declined so might have the experimental effect.

It is of particular interest that lithium affects bipolar affective disorders both by lengthening the intervals between episodes and by reducing the number of episodes per unit of time. It also reduces the severity of a given episode (Gershon and Shopsin, 1973). The present model therefore captured several distinct aspects of lithium's phenomenology and pharmacological mode of action. Not only was the normal intensity of activation reduced but its temporal structure was systematically altered in a predictable fashion. These data speak to the ability of the lithium ion to simultaneously reduce activity while altering normally occurring biological oscillations. While it was not established whether these two classes of effects are mediated by the same physiological mechanism, the present model does offer a means of further investigating this and related problems.

\section{Acknowledgements}

These data were collected while the author was supported by postdoctoral grant MH07417 from the National Institute of Mental Health. The technical assistance of $J$. Ritchie and B. Perry, and the guidance of Dr. Bernard J. Carroll are acknowledged with gratitude.

\section{References}

CARROLL, B.J. and SHARP, P.T. (1971) Rubidium and lithium opposite effects on amine mediated excitement. Science 172: 1355-1357. 
COX, C., HARRISON-READ, P.E., STEINBERG, H. and TOMKIEWICZ, M. (1971) Lithium attenuates drug induced hyperactivity in rats. Nature (Lond.) 232: $336-338$.

D' ENCARNACAO, P.S. and ANDERSON, K. (1970) Effects of lithium pretreatment on amphetamine and DMI tetrabenazine produced psychomotor behavior. Dis. Nerv. Syst. 31: 494-496.

ENGELMANN, W. (1973) A slowing down of circadian rhythms of lithium ions. 2. Naturforsch. 28: 733-736.

ENGELMANN, W., MAURER, A., MULBACH, M. and JOHNSSON, A. (1974) Action of lithium ions and heavy water in slowing circadian rhythms of petal movement in Kalanchoe. J. Interdiscipl. Cycle Res. 5: 199-205.

GERSHON, S. and SHOPSIN, B. (1973) Lithium: Its Role in Psychiatric Research and Treatment. Plenum, New York, $358 \mathrm{pp}$.

GRAY, P., SOLOMON, J., DUNPHY, M., CARR, F. and HESSION, M. (1966) Effects of lithium on open field behavior in stressed and unstressed rats. Psychopharmacology. 48: 277-281.

JOHNSON, F.N. (1972) Effects of alkali metal chlorides on activity in rats. Nature (Lond.) 238: $333-334$.

JOHNSON, F.N. (1976) Lithium effects upon components of activity in rats. Experientia. 32: 212-213.

KATZ, R.J., CARROLL, B.J. and BALDRIGHI, G. (1978) Behavioral activation by enkephalins in mice. Pharmac. Biochem. Behav. 8: 493-496.

MUKHERJEE, B.P., BAILEY, P.T. and PRADHAN, S.N. (1977) Correlation of 1 ithium effects on motor activity with its brain concentration in rats. Neuropharmacol. 16: $241-244$.

OLESEN, O.V., JENSEN, J. and THOMSEN, K. (1975) Effect of potassium on lithium induced growth retardation and polyuria in rats. Acta Pharmacol. et Toxicol. 36: 161-171.

SEGAL, D.S., CA LLAGHAN, M. and MANDELL, A.J. (1975) Alterations in behavior and catecholamine biosynthesis induced by lithium. Nature 254: 58-59.

SIEGEL, S. (1956) Nonparametric Statics for the Behavioral Sciences. McGraw Hill, New York, 312 pp.

SMITH, D.F. (1975) Biogenic amines and the effect of short term lithium administration on open field activity in rats. Psychopharmacologia. (Berl.) 30: 295-300.

SMITH, D.F. and SMITH, H. (1973) The effect of prolonged lithium administration on activity, reactivity and endurance in the rat. Psychopharmacologia (Berl.) 30: 83-88.

SYME L.A. and SYME G.J. (1973) Effects of lithium chloride on the activity of rats tested alone or in pairs. Psychopharmacologia. (Berl.) 85-89.

WOLTHIUS, O.L., DEVROOME, H. and VANWERSCH, R.A. (1975) Automatically determined effects of lithium, scopolamine, and methamphetamine on motor activity in rats. Pharmac. Biochem. Behav. 3: 515-518.

Inquiries and reprint requests should be addressed to:

Dr. R.J. Katz

Mental Health Research Institute, Department of Psychiatry

University of Michigan Medical Center, Ann Arbor, MI 48109, USA 